\title{
Outcome of surgical management of plano-valgus foot in cerebral palsy with batchelor's extra-articular subtalar arthrodesis
}

\author{
G. Jagadesh ${ }^{1}$, T. Naveen Babu ${ }^{2, *}$, S.M.Venugopal ${ }^{3}$, G. Karthik ${ }^{4}$, K. Pavan Kumar Yadav ${ }^{5}$ \\ ${ }^{1}$ Professor \& Director, ${ }^{2-4}$ Assistant Professor, ${ }^{5}$ Postgraduate, Dept. of Orthopaedics, Balaji Institute of Surgery, Research and \\ Rehabilitation for the Disabled (BIRRD) Hospital Tirupati, Andhra Pradesh, India
}

*Corresponding Author:

Email: naveen782@gmail.com

\begin{abstract}
Introduction: Foot deformities caused by altered or abnormal muscle forces are common in patients with cerebral palsy. The incidence of foot deformities in cerebral palsy is approximately $70 \%$ to $90 \%$. The most common deformity is ankle equinus, with equinovarus and equinovalgus deformities being equally common. A foot deformity can have significant effects on the patient's overall ambulatory level. The purpose of this study was to assess the functional outcome of batchelor's extra-articular subtalar arthrodesis in spastic planovalgus foot in cerebral palsy children.

Materials and Methods: This is a prospective study of 40 patients (68 feet) with spastic planovalgus foot deformity presenting to BIRRD (T) Hospital from October 2015 to October 2017. Children with spastic planovalgus foot between 6 yrs-14 yrs were included in study. There were 24 male and 16 female children. The mean age at the time of surgery was 10 yrs. Both feet were involved in 28 children, 8 had right foot involvement and 4 had left foot involvement. In our study we had 12 hemiplegics and 28 diplegics. Children with mental retardation, extra pyramidal type of cerebral palsy, rigid plano-valgus foot, spastic quadriplegia were excluded from the study. All patients underwent batchelor's arthrodesis with fibular graft and were immobilized in below knee POP cast for 8 weeks. 24 patients underwent gastrosoleus lengthening along with batchelor's arthrodesis. Clinical and functional outcome was assessed based on AOFAS (American orthopaedic foot and ankle score) clinical rating system. Radiological assessment was done by measuring Lateral talocalcaneal angle and Talar declination angle. The results of batchelor's arthrodesis are stated as satisfactory and unsatisfactory.

Satisfactory: Clinical and roentgenographic stabilization of the hind foot and no recurrence of planovalgus deformity. Unsatisfactory: Clinical and roentogenographic evidence of failure of stabilization.

Results and Discussion: All children had spastic type of cerebral palsy. Post-operatively children were followed-up for an average period of 14 months, with range from 4 to 24 months. Among 40 patients ( 68 feet), 8 feet (12 percent) had residual valgus, 12 feet (18 percent) had graft absorption, 4 feet ( 6 percent) had fracture of the graft and none had varus deformity after an average follow-up of 14 months. Out of 68 feet, 44 feet had "satisfactory" result (65 percent) and 24 feet had "unsatisfactory" result (35 percent).

Conclusion: Our experience concludes that batchelor's extra-articular arthrodesis of sub-talar joint is a good procedure for correction of plano-valgus foot deformity in cerebral palsy patients. Advantages of the procedure are simplicity of the technique, immediate firm fixation and early weight bearing.
\end{abstract}

Keywords: Cerebral palsy, Batchelor's subtalar arthrodesis, Planovalgus foot, AOFAS clinical rating system.

\section{Introduction}

Cerebral palsy is a heterogeneous disorder of movement and posture that has a wide variety of presentations, ranging from mildly affected individuals who present with some degree of motor disturbance to severely affected patients who have total body involvement. Cerebral palsy occurs in 1 to 7 children per 1000 throughout most of the world. ${ }^{1}$ In India, the occurrence is approximately 1.5 to 3.5 per 1000 live births. $^{2}$

Foot deformities caused by altered or abnormal muscle forces are common in patients with cerebral palsy, with approximately $70 \%$ to $90 \%$ of children affected. The most common deformity is ankle equinus, with equinovarus and equinovalgus deformities being equally common. Spasticity of the gastrocnemiussoleus usually is accompanied by overpull of the peroneal muscles or weakness of the foot inverters or both. Spasticity of the gastrocnemius-soleus is the primary deforming force. The contracted Achilles tendon acts as a bowstring, preventing dorsiflexion of the ankle. The dorsiflexion observed during gait occurs at the midtarsal joints, causing the calcaneus to evert and removing the sustentaculum tali from its normal supporting position underneath the talus. This, along with abduction of the midtarsal joint, causes the talus to move into a more medial and vertical position. External rotation deformity of the tibia also plays a role in this deformity. This altered talar position may cause pain with weight bearing and callus formation over the uncovered talar head. For this reason, gastrocnemiussoleus lengthening should accompany any procedure intended to correct equinovalgus. Plano-valgus foot deformity is more common in patients of spastic cerebral palsy. The condition results in functional impairment of gait for these patients and can lead to difficulties with shoe-fitting and to the development of painful plantar callosities. Most patients can be treated conservatively with shoe modifications or an orthosis to help control the hindfoot eversion. Operative treatment is indicated for patients in whom conservative treatment fails and who have significant deformity that is either 
painful or limits function. Surgical correction with subtalar arthrodesis as a single operation or combination with arthrodesis of the other midtarsal joints can be used to correct hind foot deformity, improve foot stability and relieve pain. Various techniques for performing an extra-articular subtalar arthrodesis have been described. The aim of the current study is to evaluate the clinical and functional outcome of the batchelor's extra-articular subtalar arthrodesis based on American Orthopaedic Foot and Ankle Score (AOFAS) $)^{3}$ clinical rating system and to assess the radiological outcome by measuring Lateral Talocalcaneal angle (LTCA) and Talar declination angle (TDA). ${ }^{4}$

\section{Materials and Methods}

This is a prospective study of 40 children (68 feet) with spastic planovalgus foot deformity presenting to BIRRD (T) Hospital from October 2015 to October 2017. Written informed consent was taken from the parents of all the children. Ethical committee approval was also taken.

\section{Inclusion Criteria:}

1. Children with spastic cerebral palsy.

2. Age group 6 years to 14 years.

3. Foot could be passively manipulated into corrected position.

4. Reasonable level of understanding and intelligence.

5. Ambulatory child with/without support (GMFCS Score 3)

\section{Exclusion Criteria:}

1. Mental retardation.

2. Extra pyramidal type of cerebral palsy.

3. Rigid plano-valgus foot.

4. Spastic quadriplegia.

Pre-operative Evaluation: All selected children were evaluated as per detailed history and clinical examination based on the prepared proforma. They also underwent clinical assessment done by the paediatrician to rule out other systemic involvement.

Surgical Procedure: The surgery was done under spinal anaesthesia with a pneumatic tourniquet applied over the upper thigh.

Removal of Fibular Graft: ${ }^{5}$ Three points were kept in mind while removing a fibular graft.

1. Peroneal nerve should not be damaged.

2. Distal fourth of the bone is left to maintain a stable ankle.

3. Peroneal muscles are not be cut.

Linear incision is given along the posterior border of the fibula leaving behind the distal fourth of the bone. The length of the incision must correspond to the length of the fibular graft. The plane was developed between soleus muscle posteriorly and peroneal muscles anteriorly. The dissection was deepened down to the bone. The flexor muscles and soleus from the posterior aspect and peroneal muscles from anterior aspect of the fibula was stripped in a distal to proximal direction. The neurovascular structures was protected by staying close to the bone. Linear incision was given on the bone in line with the skin incision and the periosteum was elevated. The interosseus membrane was stripped subperiosteally from proximal to distal direction. Drill holes was made proximally and distally on the fibula perpendicular to the long axis of the bone. The drill holes were joined by an osteotome and the fibular graft was removed. The fibular graft was preserved in a blood soaked gauze till it was inserted through the neck of the talus.

Exposure of Neck of the Talus: ${ }^{6}$ Neck of the talus was accessed through the anterior approach to the ankle. A longitudinal incision was made over anterior aspect of the ankle joint midway between the malleoli, ending over the dorsum of the foot. The superficial peroneal nerve was identified and protected. The extensor retinaculum was incised in line with the skin incision. The plane between the extensor hallucis longus and the extensor digitorum longus, and the neurovascular bundle between them was identified. The neurovascular bundle was retracted medially along with extensor hallucis longus. The extensor digitorum longus was retracted laterally. The joint capsule was incised longitudinally to expose the neck of talus. The anterior part of the neck of the talus was then exposed and the foot was held in the corrected position. A Krischner wire was passed across the anterior part of the talar neck in a posterior and lateral direction, crossing the talocalcaneal joint. A hole of sufficient size to accommodate the graft was drilled over the Kirschner wire using serial reamers starting from $6 \mathrm{~mm}$ diameter. The graft was then placed in the prepared hole and its distal end was embedded in the calcaneus.

Post-operative Period: A below-knee cast was applied and retained for 8 weeks. Patient was advised nonweight bearing for four weeks and partial weight bearing for another four weeks. The results of batchelor's arthrodesis was evaluated by clinical examination, functional and roentgenographic study objectively and subjectively. The results are stated as Satisfactory and Unsatisfactory. ${ }^{7}$

Satisfactory: Clinical and roentgenographic stabilization of the hind part of the foot; no recurrence of deformity.

Unsatisfactory: Clinical and roentogenographic evidence of failure of stabilization.

Weight bearing lateral roentgenograms are taken preoperatively as well as post-operatively and lateral Talocalcaneal Angle and Talar Declination Angle are measured.

Radiological Assessment: Radiological assessment was done by measuring Lateral talocalcaneal angle (LTCA) and Talar Declination Angle (TDA).

Lateral Talo-calcaneal Angle(LTCA): It is the angle formed between the calcaneal axis (CA) and the Collum 
Tali Axis (CTA). Normal lateral talocalcaneal angle: 25-50 degrees.

Talar Declination Angle (TDA): It is the angle formed between the plane of support and the collum tali axis.

Normal talar declination angle: 15-55 degrees.

Collum Tali Axis (CTA): It is a line bisecting the head and neck of the talus.

Plane of Support (PS): It is a line connecting the most inferior point of the tuberosity of the calcaneus to the most inferior point of the fifth metatarsal head.

\section{Results and Discussion}

The results of batchelor's arthrodesis was evaluated by clinical examination, functional and roentgenographic study objectively and subjectively. The results are stated as "satisfactory" and "unsatisfactory". A rating of satisfactory indicated extra-articular arthrodesis of the subtalar joint clinically as well as incorporation of the graft roentgenographically at the last examination, maintenance of correction of the deformity with adequate reduction of the calcaneus under the talus, and an asymptomatic foot with no clinical valgus or varus deviation of either the hind or the fore part of the foot. If any of these criteria were not met the result was rated unsatisfactory.

Satisfactory: Clinical and roentgenographic stabilization of the hind part of the foot; no recurrence of deformity.

Unsatisfactory: Clinical and roentogenographic evidence of failure of stabilization.

Clinical and functional outcome was assessed based on AOFAS (American orthopaedic foot and ankle score) clinical rating system. Radiological assessment was done by measuring Lateral Talocalcaneal Angle (LTCA) and Talar Declination Angle (TDA). Weight bearing lateral roentgenograms are taken preoperatively as well as post-operatively. This is a prospective study of 40 children (68 feet) with planovalgus foot deformity in cerebral palsy presenting to BIRRD (T) Hospital from October 2015 to October 2017. Children with spastic planovalgus foot between 6 yrs-14 yrs were included in study. There were 24 male and 16 female children. The mean age at the time of surgery was 10 yrs. Both feet were involved in 28 children, 8 had right foot involvement and 4 had left foot involvement. In our study we had 12 hemiplegics and 28 diplegics. Children with mental retardation, extra pyramidal type of cerebral palsy, rigid plano-valgus foot, spastic quadriplegia were excluded from the study. All patients underwent batchelor's arthrodesis with fibular graft and were immobilized in below knee POP cast for 8 weeks. 24 patients underwent gastrosoleus lengthening along with batchelor's arthrodesis. Post-operatively children were followed-up for an average period of 14 months, with range from 4 to 24 months. Out of 40 children, 24 children had a total pre-operative AOFAS score of 54, it was improved to 79 post-operatively. 10 children had a total pre-operative AOFAS score of 71 preoperatively, it was improved to 94 post-operatively. Remaining 6 children had a pre-operative score of 88 pre-operatively, it was improved to 94 postoperatively. The p-value of 0.001 was obtained for the total score of AOFAS clinical rating system which indicated a highly significant relation. The pre-operative mean values of LTCA and TDA was 44 and 38 degrees respectively. Post-operative values of LTCA and TDA values was 32 and 28 degrees respectively. The angles at final followup was 30 and 26 degrees. The mean improvement was 12 degrees. The LTCA and TDA were maintained within normal limits after an average follow-up of 14 months. Among 40 patients (68 feet), 8 feet (12 percent) had residual valgus, 12 feet (18 percent) had graft absorption, 4 feet ( 6 percent) had fracture of the graft and none had varus deformity after an average follow-up of 14 months. Out of 68 feet, 44 feet had "satisfactory" result (65 percent) and 24 feet had "unsatisfactory" result (35 percent).

Table 1: Total AOFAS score

\begin{tabular}{|l|c|c|}
\hline $\begin{array}{l}\text { No. of } \\
\text { children }\end{array}$ & $\begin{array}{c}\text { Pre-op } \\
\text { score }\end{array}$ & $\begin{array}{c}\text { Post-op } \\
\text { score }\end{array}$ \\
\hline 6 & 88 & 94 \\
\hline 10 & 71 & 94 \\
\hline 24 & 54 & 79 \\
\hline
\end{tabular}

Table 2: Radiological findings (mean, range) in degrees:

\begin{tabular}{|l|c|c|}
\hline & $\begin{array}{c}\text { Lateral } \\
\text { talocalcaneal } \\
\text { angle } \\
\text { (LTCA) }\end{array}$ & $\begin{array}{c}\text { Talar } \\
\text { declination } \\
\text { angle (TDA) }\end{array}$ \\
\hline Pre-operative & $44(32$ to 56) & $38(25$ to 52) \\
\hline $\begin{array}{l}\text { Post- } \\
\text { operative }\end{array}$ & $32(21$ to 44) & $28(20$ to 36) \\
\hline $\begin{array}{l}\text { Final follow- } \\
\text { up }\end{array}$ & $30(20$ to 44) & $26(19$ to 32) \\
\hline
\end{tabular}

Table 3: Complications

\begin{tabular}{|l|c|}
\hline Complication & No. of feet \\
\hline Residual valgus & 8 \\
\hline Graft absorption & 12 \\
\hline Fracture of the graft & 4 \\
\hline Varus deformity & 0 \\
\hline
\end{tabular}

Table 4: Results

\begin{tabular}{|l|l|}
\hline Satisfactory & 44 feet \\
\hline Unsatisfactory & 24 feet \\
\hline
\end{tabular}




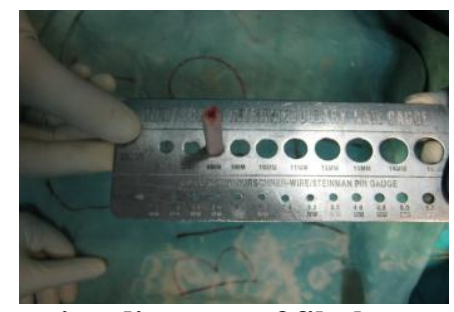

Fig. 1: Measuring diameter of fibular graft

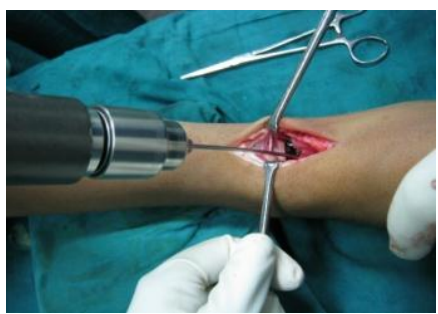

Fig. 2: K-wire passed through neck of the talus into the calcaneum

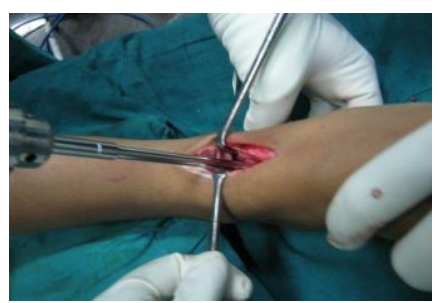

Fig. 3: Serial reaming to accommodate the fibular graft
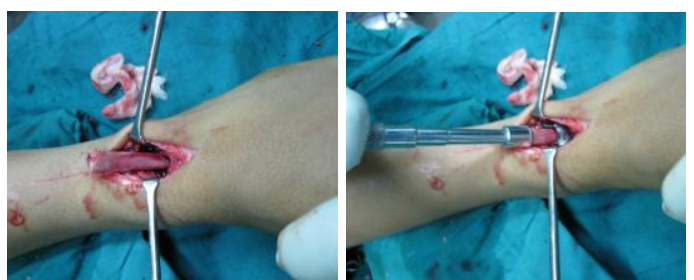

Fig. 4: Insertion of fibular graft through talar neck into the calcaneum

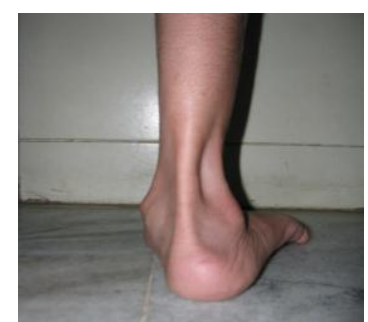

Fig. 5: Pre-op photograph

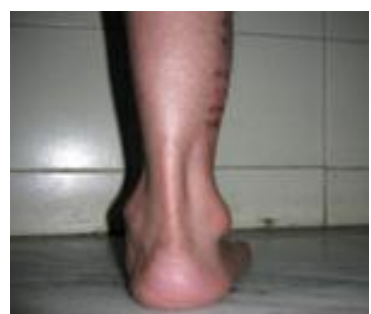

Fig. 6: Post-operative photograph

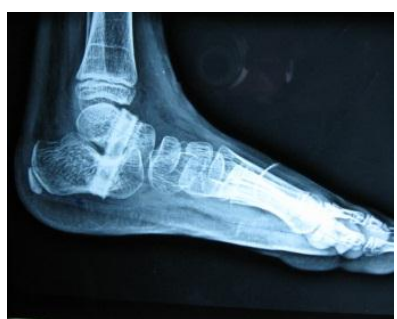

Fig. 7: Post-operative radiograph: graft in position with extra articular sub-talar joint fusion

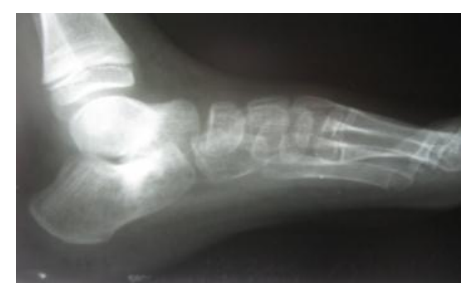

Fig. 8: Radiograph showing graft resorption

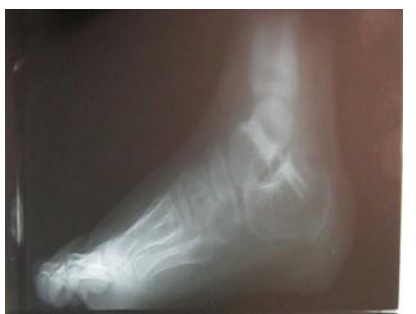

Fig. 9: Radiograph showing graft fracture
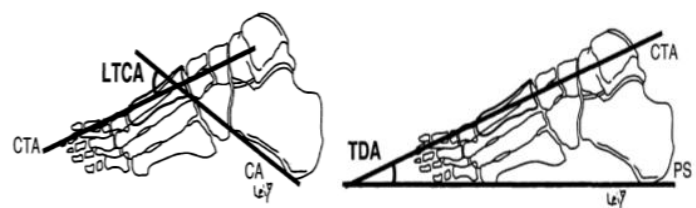

Fig. 10: Images depicting lateral talocalcaneal angle (LTCA) and talar declination angle (TDA)

\section{Conclusion}

Our experience concludes that batchelor's extraarticular arthrodesis of sub-talar joint is a satisfactory procedure for correction of plano-valgus foot deformity in cerebral palsy children. Advantages of the procedure are simplicity of the technique, immediate firm fixation and early weight bearing. 40 children (68 feet) with plano-valgus foot deformity in cerebral palsy were analyzed with an average follow-up of 14 months. Diplegia was the most common type of cerebral palsy in our study. Spastic type was the most common physiological type. Radiological assessment was done by measuring Lateral talocalcaneal angle (LTCA) and Talar declination angle (TDA). The angles were maintained within normal limits at an average followup of 14 months. Out of 68 feet operated, 8 feet (12 percent) had residual valgus, 12 feet (18 percent) had graft absorption, 4 feet (6 percent) had fracture of the graft and none had varus deformity after an average follow-up of 14 months. Out of 68 feet, 44 feet had 
"satisfactory" result (65 percent) and 24 feet had "unsatisfactory" result (35 percent). Clinical and functional assessment was done by AOFAS clinical rating system.Children had improved in their functional activities, walking distance, walking surfaces, gait, alignment and stability. The p-value of 0.001 was obtained for the total score of AOFAS clinical rating system which indicated a highly significant relation. Large scale study with long term follow up is required to corroborate findings of the study and to find out long term functional results

\section{References}

1. Nonica Laisram, V. K. Srivastava and R. K. Srivastava: Cerebral palsy-An etiological study. Indian Journal of Pediatrics, Volume 59; Number 6:723-728.

2. Loder RT: Orthopaedic aspects of children with infectious (central nervous system) postnatal cerebral palsy. J Pediatric Orthop 1992;12:527.

3 . Kitaoka et al, Clinical rating systems for the anklehindfoot, midfoot, hallux and lesser toes-Foot and ankle international. Vol.15.no.7 July 1994.

4. A.Gentili et al, Pictorial review: Foot axes and angles; the British journal of radiology 1996;69,968-974.

5. Hoppenfeld, Stanley, de Boer, Piet, Surgical exposures in orthopaedics-the anatomic approach vol.3 chapter 11.

6. Hoppenfeld, Stanley, de Boer, Piet, Surgical exposures in orthopaedics-the anatomic approach vol.3 chapter 12 .

7. L.C.S. Hsu et al- Batchelor's extra-articular subtalar arthrodesis- J Bone Joint Surg vol.58-A no.2 march 1976. 